\title{
Hemisphere-specific optogenetic stimulation reveals left-right asymmetry of hippocampal plasticity
}

\author{
Michael M. Kohl' 1,2 , Olivia A. Shipton ${ }^{1,2}$, Robert M. Deacon ${ }^{2,3}$, J. Nicholas P. Rawlins ${ }^{2,3}$, \\ Karl Deisseroth ${ }^{4}$, and Ole Paulsen ${ }^{1,2}$ \\ ${ }^{1}$ Department of Physiology, Development and Neuroscience, University of Cambridge, Downing \\ Street, Cambridge CB2 3EG, UK \\ ${ }^{2} \mathrm{OXION}$ initiative, Department of Physiology, Anatomy and Genetics, University of Oxford, Parks \\ Road, Oxford OX1 3PT, UK \\ ${ }^{3}$ Department of Experimental Psychology, University of Oxford, Oxford OX1 3UD, UK \\ ${ }^{4}$ Department of Bioengineering, Stanford University, Stanford, CA 94305, USA
}

\begin{abstract}
Postsynaptic spines at CA3-CA1 synapses differ in glutamate receptor composition according to the hemispheric origin of CA3 afferents. To study the resulting functional consequences, we used optogenetic tools to selectively stimulate axons of CA3 pyramidal cells originating in either left or right mouse hippocampus. We found that left $\mathrm{CA} 3$ input produced more long-term potentiation at CA1 synapses than right CA3 input, due to differential expression of GluN2B subunit-containing NMDA receptors.
\end{abstract}

\section{Keywords}

STDP; LTP; Channelrhodopsin-2; hippocampus; mouse

\begin{abstract}
Hemispheric brain asymmetry has been the focus of numerous anatomical and psychological studies $^{1}$, and possible molecular correlates of such asymmetry have recently been identified in the mouse hippocampus ${ }^{2-4}$. It was found that postsynaptic spines of CA1 pyramidal cells targeted by projections from the right CA3 are larger and have higher GluA1, but lower GluN2B, expression than postsynaptic spines contacted by projections from the left CA3 (ref 3). Since changes in glutamate receptor distribution are thought to mediate long-term synaptic plasticity ${ }^{5}$, we sought to investigate the effect of this hemispheric asymmetry on spike timing-dependent long-term potentiation (t-LTP) $)^{6,7}$.

In order to selectively stimulate axons from either left or right CA3 pyramidal neurons, we made stereotactic injections of an adeno-associated virus containing double-inverse floxed Channelrhodopsin2-eYFP ${ }^{8}$ (Fig. 1a) into either the left or right CA3 region in adult CamKIIa::cre transgenic mice. This led to Cre-dependent, selective expression of Channelrhodopsin2-eYFP in CamKIIa-positive cells (i.e. excitatory cells) in the CA3 of one hippocampus and their ipsilateral and contralateral projections (Schaffer collateral and commissural fibers, respectively) (Fig. 1b,c). Fourteen to twenty-eight days after injection,
\end{abstract}

Address for correspondence: Professor Ole Paulsen, op210@cam.ac.uk, University of Cambridge, Department of Physiology, Development and Neuroscience, Physiological Laboratory, Downing Street, Cambridge, CB2 3EG, UK.

Author Contributions: M.M.K. conducted the experiments and analyzed the data. O.A.S. contributed recordings. M.M.K. and R.M.D. injected the animals. J.N.P.R. provided advice on the project. K.D. designed and cloned the AAV DIO ChR2-YFP vector. M.M.K. and O.P. designed the experiments. M.M.K., O.A.S. and O.P. wrote the manuscript. All authors approved the manuscript. 
we prepared coronal slices of the hippocampal formation and obtained whole-cell recordings from CA1 pyramidal cells (Supplementary Methods online). Optical stimulation with 473 $\mathrm{nm}$ laser light in the stratum radiatum evoked reliable excitatory postsynaptic potentials (EPSPs) over the course of the experiment (EPSP slope remained stable for more than 50 minutes at $0.1 \mathrm{~Hz}$ stimulation, $\mathrm{n}=5$, Supplementary Fig. 1). These responses were comparable to EPSPs evoked by extracellular electrical stimulation, in that both were mediated by the generation of axonal action potentials (>99\% EPSP block by $1 \mu \mathrm{M}$ tetrodotoxin, $\mathrm{n}=4$; Fig. 1d) and showed similar paired-pulse ratios $\left(\mathrm{PPR}_{\text {electrical }}: 2.0 \pm 0.1\right.$, $\mathrm{n}=18 ; \mathrm{PPR}_{\text {optical }}: 1.8 \pm 0.2, \mathrm{n}=17$; Fig. 1e). Optical and electrical stimulation consistently recruited independent inputs since no cross-facilitation was observed (PPR electrical-optical 1.1 $\pm 0.1, \mathrm{n}=6$; $\mathrm{PPR}_{\text {optical-electrical }}: 1.1 \pm 0.2, \mathrm{n}=6$; Fig. 1e)

We next investigated t-LTP at the CA3-CA1 synapse, monitoring EPSPs evoked by alternating optical and electrical stimulation in the stratum radiatum. Whereas electrical stimulation activates fibers irrespective of their hemispheric origin, optical stimulation specifically recruits fibers from the injected CA3. To guard against possible effects of different expression patterns or levels following left and right injection, t-LTP was induced under equivalent conditions by adjusting light intensity and electrical current to produce similar sized EPSPs $(2-4 \mathrm{mV})$, and using a burst pairing protocol ${ }^{7}$ with simultaneous activation of electrical and optical input. Pairing consisted of presynaptic stimulation followed 5-10 ms later by a postsynaptic burst of action potentials and was repeated 100 times (Supplementary Methods online). There was a significant difference in the magnitude of t-LTP induced depending on the hemispheric origin of afferent fibers. In slices prepared from left-injected animals, pairing elicited robust t-LTP in the optically-evoked input (144 \pm $8 \%, \mathrm{n}=14 ; \mathrm{p}<0.01$, Student's $t$-test), whereas slices from right-injected animals showed no $\mathrm{t}$-LTP in the optical pathway $\left(93 \pm 8 \%, \mathrm{n}=14\right.$; n.s.). Consistent with earlier reports ${ }^{9}, 10$, this was not caused by possible differences between ipsilateral and contralateral pathways, as we found t-LTP of equivalent magnitude in the ipsilateral and contralateral optical pathway in slices from left-injected mice (ipsi, $145 \pm 9 \%, \mathrm{n}=7$; contra, $142 \pm 13 \%, \mathrm{n}=7$; Fig. 2a-c). In contrast, we found no t-LTP in either optical pathway in right-injected mice (ipsi, $96 \pm 10 \%$, $\mathrm{n}=7$; contra, $90 \pm 12 \%, \mathrm{n}=7$; Fig. 2d-f). There were no significant differences between the electrical pathways (left-injected: ipsi, $164 \pm 12 \%, \mathrm{n}=7$; contra, $160 \pm 8 \%, \mathrm{n}=7$; rightinjected: ipsi: $159 \pm 12 \%, \mathrm{n}=7$; contra: $163 \pm 14 \%, \mathrm{n}=7$ ).

To exclude the possibility that this left-right difference was due to an induction threshold effect, we repeated the experiments with a stronger pairing protocol in the hippocampus contralateral to the injection side and the experimenter blind to injection side, producing equivalent results (optical pathway: left-injected, $142 \pm 20 \%, \mathrm{n}=6$; right-injected, $111 \pm$ $12 \%, \mathrm{n}=6$; electrical pathway: left-injected, $150 \pm 15 \%, \mathrm{n}=6$; right-injected, $169 \pm 19 \%, \mathrm{n}$ =6; Supplementary Fig. 2).

Subsequently, we wanted to examine a possible mechanism underlying this asymmetry in tLTP. NMDA receptor (NMDAR) activation is known to be required for induction of $\mathrm{LTP}^{6}$; thus we first tested whether CA1 cells showed a difference in NMDA/AMPA receptormediated current ratio (NMDA/AMPA ratio) depending on the hemispheric origin of their presynaptic input from CA3. However, there was not: the overall NMDA/AMPA ratio was not different with respect to input (left-injected: optical stimulation, $23 \pm 3 \%$; electrical stimulation, $26 \pm 4 \%$; right-injected: optical, $27 \pm 3 \%$, electrical, $25 \pm 2 \%, \mathrm{n}=6$; Fig. 3 a). Nevertheless, there was an NMDAR subunit-dependent difference between the two sides: the selective GluN2B subunit antagonist Ro 25-6981 $(0.5 \mu \mathrm{M})$ blocked $60 \%$ of the total NMDAR current from left CA3 inputs compared to $38 \%$ of the total NMDAR current from right CA3 inputs (left-injected: Ro 25-6981, $22 \pm 1$ pA versus control, $55 \pm 9$ pA, $\mathrm{n}=6$; right-injected: Ro 25-6981, $33 \pm 3$ pA versus control, $53 \pm 7$ pA; n = 7; Fig. 3b). To test 
whether this difference could explain why left CA3 input displays more t-LTP, we repeated pairing experiments in the presence of $0.5 \mu \mathrm{M}$ Ro 25-6981. t-LTP in left-injected animals was completely blocked under these conditions (Fig. 3c), suggesting the difference in GluN2B subunit expression is sufficient to explain the hemispheric asymmetry in t-LTP. Similar to electrical pairing-induced t-LTP, the optically-evoked t-LTP is most likely expressed postsynaptically, as we observed no change in paired-pulse ratios following t-LTP induction (Supplementary Table 1).

In summary, using optogenetic tools for cell-type- and hemisphere-specific recruitment of CA3 axons, we have shown that inputs from the left CA3 onto CA1 pyramidal cells are more able to produce t-LTP than inputs from the right CA3. This hemispheric asymmetry in plasticity could be explained by differential GluN2B expression at synapses targeted by the left compared to right CA3 (ref. 3), consistent with recent evidence suggesting that GluN2B subunit-containing NMDARs favor LTP induction ${ }^{11,12}$. Since cortical NMDAR subunit expression appears to be regulated by activity-dependent mechanisms ${ }^{12-14}$, this result raises the intriguing possibility that the left and right $\mathrm{CA} 3$ might be differentially active and hence produce input-specific differences in postsynaptic spines, consistent with recent morphological observations that spines receiving input from right $\mathrm{CA} 3$ are more mature than those receiving left CA3 input (ref. 3). The behavioral consequences of the hemispherespecific asymmetry in synaptic plasticity we have uncovered here remain to be investigated, but such experiments are now possible using optogenetic control of neuronal activity in vivo ${ }^{15}$.

\section{Supplementary Material}

Refer to Web version on PubMed Central for supplementary material.

\section{Acknowledgments}

This research was supported by the BBSRC and the Wellcome Trust. An equipment grant from the EPA Cephalosporin Fund is gratefully acknowledged. The authors would like to thank David Paterson for providing surgery facilities and Dennis Kätzel and Louise Upton for help with immunohistochemistry.

\section{References}

1. Toga AW, Thompson PM. Nat. Rev. Neurosci. 2003; 4:37-48. [PubMed: 12511860]

2. Wu Y, et al. J. Neurosci. 2005; 25:9213-9226. [PubMed: 16207881]

3. Shinohara Y, et al. Proc. Natl. Acad. Sci. USA. 19498; 2008; 105

4. Kawakami R, et al. Science. 2003; 300:990-994. [PubMed: 12738868]

5. Malinow R, Malenka RC. Annu. Rev. Neurosci. 2002; 25:103-126. [PubMed: 12052905]

6. Bliss TV, Collingridge GL. Nature. 1993; 361:31-39. [PubMed: 8421494]

7. Pike FG, Meredith RM, Olding AW, Paulsen OJ. Physiol. 1999; 518:571-576.

8. Sohal VS, Zhang F, Yizhar O, Deisseroth K. Nature. 2009; 459:698-702. [PubMed: 19396159]

9. Bliss TVP, Lancaster B, Wheal HV. J. Physiol. 1983; 341:617-626. [PubMed: 6620191]

10. Wheal HV, Lancaster B, Bliss TVP. Brain Res. 1983; 272:247-253. [PubMed: 6311340]

11. Barria A, Malinow R. Neuron. 2005; 48:289-301. [PubMed: 16242409]

12. Yashiro K, Philpot BD. Neuropharmacology. 2008; 55:1081-1094. [PubMed: 18755202]

13. Hoffman H, Gremme T, Hatt H, Gottman KJ. Neurochem. 2000; 75:1590-1599.

14. Mierau SB, Meredith RM, Upton AL, Paulsen O. Proc. Natl. Acad. Sci. USA. 2004; 101:1551815523. [PubMed: 15492224]

15. Aravanis AM, et al. J. Neural. Eng. 2007; 4:S143-S156. [PubMed: 17873414] 

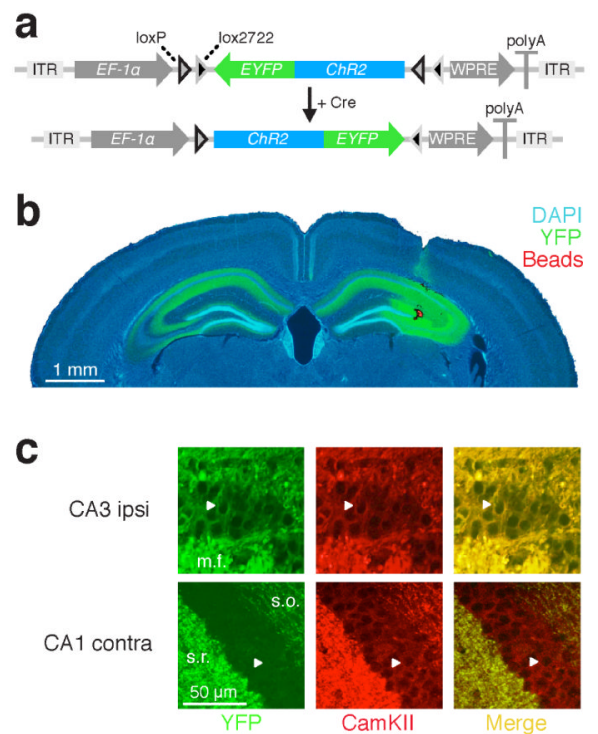

d

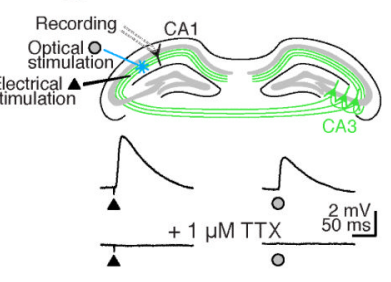

e

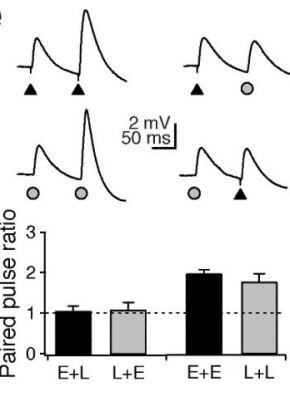

Figure 1. Optogenetics enables selective stimulation of CA3 afferents ex vivo (a) Adeno-associated virus (AAV) containing DIO ChR2-eYFP was stereotactically injected into either the left or right dorsal CA3 region of CamKIIa ::cre mice. (b) ChR2-eYFP expression was limited to the injection site (identified by co-injection of red latex beads) in the CA3 region and ipsilateral and contralateral projections. (c) Only pyramidal neurons (arrowheads) in the ipsilateral CA3 region expressed ChR2-eYFP (m.f. mossy fibers, s.r. stratum radiatum, s.o. stratum oriens). (d) Electrical and optical stimulation in the stratum radiatum produced EPSPs in CA1 pyramidal cells by evoking action potentials that were blocked by $1 \mu \mathrm{M}$ TTX. (e) Both electrical and optical stimulation displayed paired-pulse facilitation. The lack of cross-facilitation confirmed that both inputs were independent. Error bars are s.e.m. 
a
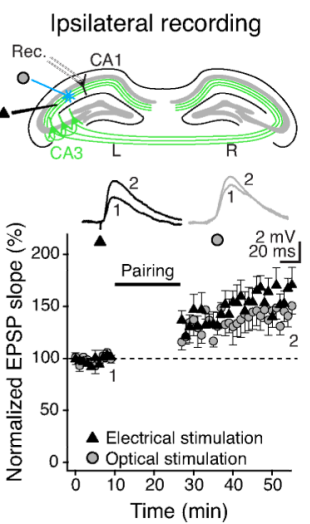

d
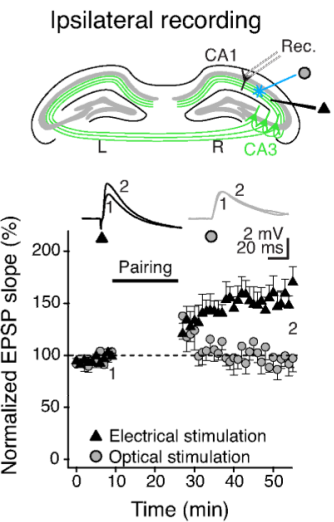

b

Contralateral recording
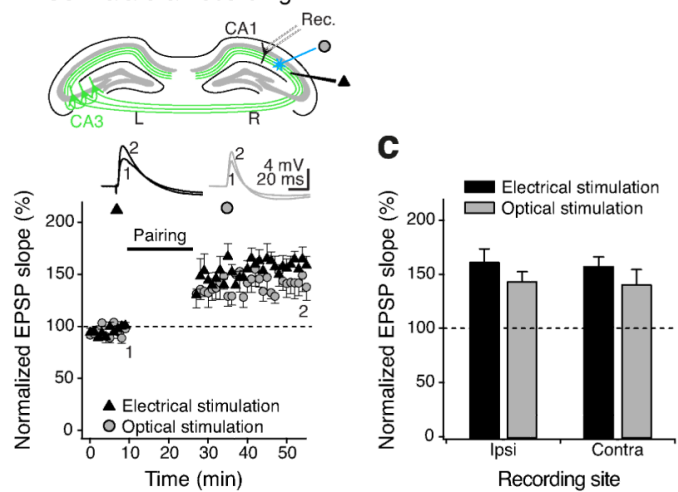

e

Contralateral recording
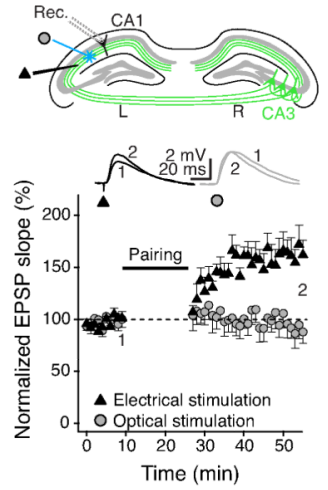

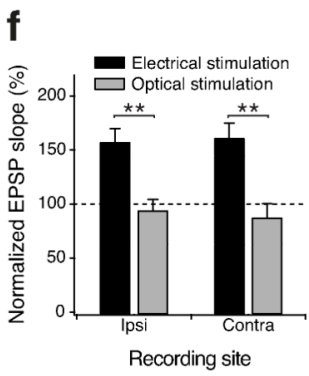

Figure 2. Hemispheric asymmetry of t-LTP at the CA3-CA1 pyramidal cell synapse (a-f) Indiscriminate electrical stimulation (triangles) in the stratum radiatum produced robust t-LTP in CA1 pyramidal neurons. (a-c) Selective optical stimulation (circles) of CA3 Schaffer collaterals (ipsilateral projections) and commissural fibers (contralateral projections) originating in the left hemisphere also both induced t-LTP. (e-f) In contrast, optical stimulation of CA3 projections originating in the right hemisphere led to significantly less t-LTP than electrical stimulation. Insets show representative EPSPs at the indicated time points $(1,2)$. Error bars are s.e.m. ${ }^{*} * \mathrm{P}<0.01$, Student's $t$-test. 

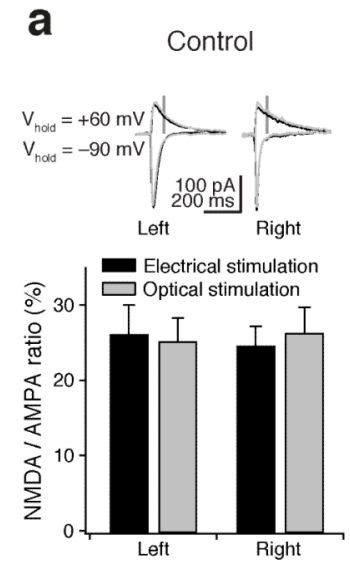

Injected hemisphere
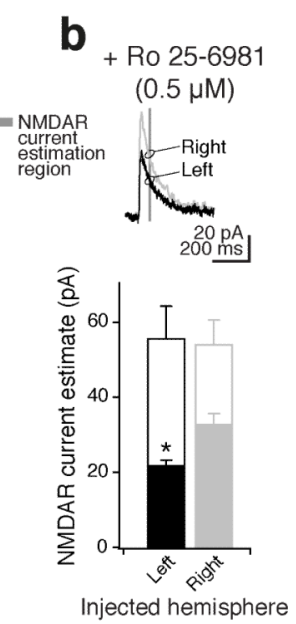

C $\quad+$ Ro $25-6981$

$(0.5 \mu \mathrm{M})$
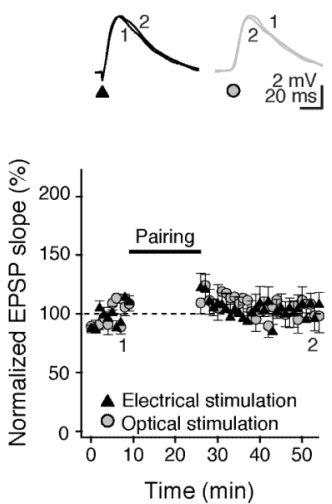

Figure 3. Asymmetric expression of GluN2B-containing NMDA receptors underlies hemispheric differences in t-LTP

(a) Hemisphere-selective optical stimulation (gray traces, gray bars) and hemisphereindiscriminate electrical stimulation (black traces, black bars) of afferents from CA3 were used to evoke postsynaptic currents in CA1 pyramidal cells contralateral to the injection side. There was no difference in the overall NMDA to AMPA receptor ratios between left and right for either electrical or optical stimulation (dark gray box indicates the time window for estimation of the NMDAR current). (b) Selective block of GluN2B subunit-containing NMDARs with 0.5 $\mu \mathrm{M}$ Ro 25-6981 affected the NMDAR current evoked by left CA3 input more than right $\mathrm{CA} 3$ input. Open bars show NMDAR current estimate in control, filled bars show remaining NMDAR current in the presence of $0.5 \mu \mathrm{M}$ Ro 25-6981. Traces show representative optically-evoked postsynaptic currents at $+60 \mathrm{mV}$ in the presence of $0.5 \mu \mathrm{M}$ Ro 25-6981 for left- and right-injected animals. (c) 0.5 $\mu \mathrm{M}$ Ro 25-6981 completely blocked t-LTP in CA1 cells receiving left CA3 (optical stimulation, circles) or mixed CA3 inputs (electrical stimulation, triangles). Insets show representative EPSPs at the indicated time points $(1,2)$. Error bars are s.e.m. $* \mathrm{P}<0.05$, Student's $t$-test. 